\title{
Efficient Frequency Estimation of a Single Real Tone based on Principal Singular Value Decomposition
}

\author{
H. C. So, Frankie K. W. Chan, Weize Sun \\ Department of Electronic Engineering, City University of Hong Kong Tat Chee Avenue, \\ Kowloon, Hong Kong
}

\begin{abstract}
The problem of single-tone frequency estimation for a discrete-time real sinusoid in white Gaussian noise is addressed. We first show that the frequency information is embedded in the principal singular vectors of a matrix which stores the observed data with no repeated entry. The technique of weighted least squares is then utilized for finding the frequency from the singular vectors. It is proved that the variance of the frequency estimate approaches Cramér-Rao lower bound when the data observation length tends to infinity. The computational efficiency and estimation accuracy are demonstrated via computer simulations.
\end{abstract}

Keywords:

Frequency estimation, single-tone, sinusoid, singular value decomposition, weighted least square

\section{Introduction}

Frequency estimation of a single real sinusoid in the presence of noise has been an active research topic in the signal processing literature [1]-[13]. The signal model is:

$\begin{aligned} r_{k} & =s_{k}+q_{k}, \quad k \\ \text { where } & s_{k}=\gamma \sin (\omega k+\phi)\end{aligned}$

The $\gamma>0, \omega \in(0, \pi)$ and $\phi \in[0,2 \pi)$ are the amplitude, frequency and phase of the sinusoid, respectively, and they are unknown constants, while $q_{k}$ is the additive measurement noise which is assumed a zero-mean white Gaussian 
process with unknown variance of $\sigma^{2}$. The task of frequency estimation is to find $\omega$ from the $K$ samples of $r_{k}$.

Pisarenko harmonic decomposition (PHD) [3] can be considered as the simplest subspace method which utilizes the covariance of $r_{k}$ to compute the frequency. So and Chan [4] have shown that the PHD estimation performance can be improved if the data matrix is employed instead of the covariance matrix. By incorporating the technique of weighted least squares (WLS) into [4], the resultant algorithm [5] can provide optimum accuracy. Recently, Elasmi-Ksibi et al. [6] have also extended [3] with the use of a normalized second-order infinite impulse response notch filter, whose frequency variance can attain Cramér-Rao lower bound (CRLB) [1] when $\omega$ is close to $0.5 \pi$. On the other hand, a fast efficient technique based on iterative autoregressive moving average (ARMA) model fitting has been devised in [7]. Maximum likelihood (ML) frequency estimation of a real tone has been addressed in [8] which involves maximizing a multimodal cost function, and this is similar to the periodogram methodology [9]. Furthermore, approaches based on discrete Fourier transform (DFT) and autocorrelation of $r_{k}$ are found in [10]-[11] and [12]-[13], respectively. Although single-tone frequency estimation has been well studied, efforts have continually been made to devise fast and accurate estimators with small threshold signal-to-noise ratio (SNR) and uniform estimation performance across the admissible frequency range. In this work, we propose to exploit the principal-singular-vector utilization for modal analysis (PUMA) [14] which is originally derived for two-dimensional sinusoidal parameter estimation, and WLS [5], in the frequency estimator development, in order to fulfil the above-mentioned challenges.

The rest of the paper is organized as follows. In Section 2, the proposed frequency estimator is derived and analyzed, assuming that $K$ can be factorized as $K=M \times N$. We first show that the frequency information is contained in the principal singular vectors of the $M \times N$ matrix which stores $r_{k}$ with no repeated entry. To be precise, the left principal singular vectors correspond to noisy sinusoidal sequences with frequency of $\omega$ while the frequency of the right principal singular vectors is $M \omega$. We then utilize the WLS technique [5] to find the frequencies of the principal singular vectors. It is also proved that when $K$ tends to infinity, the variance of the frequency estimate approaches CRLB. It is worthy to point out that we have recently applied the PUMA methodology in developing an efficient estimator for a single complex tone [15]. Nevertheless, the factorization of a real tone is different from that of a complex tone and hence the algorithm and analy- 
sis are different from [15]. Simulation results are included in Section 3 to corroborate the analytical development and to evaluate the performance of the proposed algorithm by comparing with the WLS [5], ML [8], and ARMA model fitting [7] methods as well as CRLB. Finally, conclusions are drawn in Section 4.

\section{Proposed Frequency Estimator}

We first construct a $M \times N$ matrix, denoted by $\mathbf{R}$, to store $r_{k}$ with no repeated entry, and it has the form of:

$$
\mathbf{R}=\left[\begin{array}{cccc}
r_{1} & r_{M+1} & \cdots & r_{M(N-1)+1} \\
r_{2} & r_{M+2} & \cdots & r_{M(N-1)+2} \\
\vdots & \vdots & \ddots & \vdots \\
r_{M} & r_{2 M} & \cdots & r_{M N}
\end{array}\right]
$$

The $(m, n)$ entry of $\mathbf{R}$ is represented as $[\mathbf{R}]_{m, n}=r_{m+(n-1) M}$. Without loss of generality, it is assumed that $M \geq N$. Note that even if $K$ is not factorizable, one simple way is to discard a few samples and find $M$ and $N$ such that their product is closest to $K$, and the performance loss will be negligible for a sufficiently large data length. In matrix form, (1) becomes

$$
\mathbf{R}=\mathbf{S}+\mathbf{Q}
$$

where $\mathbf{S}$ and $\mathbf{Q}$ contain $\left\{s_{k}\right\}$ and $\left\{q_{k}\right\}$ accordingly. By making use of trigonometric identities, it is found that $\mathbf{S}$ can be factorized as:

where

$$
\begin{gathered}
\mathbf{S}=\gamma \mathbf{G} \mathbf{H}^{T}=\mathbf{G} \boldsymbol{\Gamma} \mathbf{H}^{T} \\
\boldsymbol{\Gamma}=\operatorname{diag}(\gamma, \gamma) \\
\mathbf{G}=\left[\begin{array}{cccc}
\sin (\omega+\phi) & \sin (2 \omega+\phi) & \cdots & \sin (M \omega+\phi) \\
\cos (\omega+\phi) & \cos (2 \omega+\phi) & \cdots & \cos (M \omega+\phi)
\end{array}\right]^{T}
\end{gathered}
$$

and

$$
\mathbf{H}=\left[\begin{array}{llll}
\cos (0) & \cos (M \omega) & \cdots & \cos (M(N-1) \omega) \\
\sin (0) & \sin (M \omega) & \cdots & \sin (M(N-1) \omega)
\end{array}\right]^{T}
$$

with ${ }^{T}$ denotes the transpose operator. We see that the frequency information is contained in $\mathbf{G}$ and $\mathbf{H}$ but they cannot be straightforwardly obtained 
from $\left\{r_{k}\right\}$. In this work, we make use of the PUMA approach [14] by employing the principal singular vectors of $\mathbf{R}$ for frequency estimation as follows. Decomposing $\mathbf{R}$ using singular value decomposition yields:

$$
\mathbf{R}=\mathbf{U} \Lambda \mathbf{V}^{T}
$$

where $\boldsymbol{\Lambda}=\operatorname{diag}\left(\lambda_{1}, \lambda_{2}, \cdots, \lambda_{N}\right)$ is the diagonal matrix of singular values with $\lambda_{1} \geq \lambda_{2} \geq \cdots=\lambda_{N} \geq 0$ while $\mathbf{U}=\left[\begin{array}{llll}\mathbf{u}_{1} & \mathbf{u}_{2} & \cdots & \mathbf{u}_{M}\end{array}\right]$ and $\mathbf{V}=$ $\left[\begin{array}{llll}\mathbf{v}_{1} & \mathbf{v}_{2} & \cdots & \mathbf{v}_{N}\end{array}\right]$ are orthonormal matrices whose columns are the corresponding left and right singular vectors, respectively. By noting that $\operatorname{rank}(\mathbf{S})=2$ as long as $M \omega$ is not an integral multiple of $2 \pi$, the optimum estimate of $\mathbf{S}$ based on $\mathbf{R}$, denoted by $\hat{\mathbf{S}}$, is

$$
\hat{\mathbf{S}}=\mathbf{U}_{s} \Lambda_{s} \mathbf{V}_{s}^{T}
$$

where $\mathbf{U}_{s}=\left[\begin{array}{ll}\mathbf{u}_{1} & \mathbf{u}_{2}\end{array}\right], \boldsymbol{\Lambda}_{s}=\operatorname{diag}\left(\lambda_{1}, \lambda_{2}\right)$ and $\mathbf{V}_{s}=\left[\begin{array}{ll}\mathbf{v}_{1} & \mathbf{v}_{2}\end{array}\right]$ are the signal subspace components.

Comparing (5)-(8) and (10), it is clear that $\mathbf{G}, \boldsymbol{\Gamma}$ and $\mathbf{H}$ correspond to $\mathbf{U}_{s}, \boldsymbol{\Lambda}_{s}$ and $\mathbf{V}_{s}$, respectively. To estimate $\omega$ from $\mathbf{U}_{s}$, we utilize the fact that $\mathbf{G}$ and the noise-free version of $\mathbf{U}_{s}$, denoted by $\tilde{\mathbf{U}}_{s}$, span the same subspace, that is:

$$
\tilde{\mathbf{U}}_{s}=\mathbf{G} \Omega_{G}
$$

where $\Omega_{G} \in \mathbb{R}^{2 \times 2}$ is an unknown rotation matrix. As a result, $\tilde{\mathbf{u}}_{1}$ and $\tilde{\mathbf{u}}_{2}$ are still pure real tone sequences although their amplitudes and phases are different from those of $\mathbf{G}$. As an illustration, considering $K \rightarrow \infty$ and noting that the singular vectors are orthogonal and of unity norms, we can deduce:

$$
\tilde{\mathbf{U}}_{s}=\sqrt{\frac{2}{M}}\left[\begin{array}{llll}
\sin (\omega+\phi-\theta) & \sin (2 \omega+\phi-\theta) & \cdots & \sin (M \omega+\phi-\theta) \\
\cos (\omega+\phi-\theta) & \cos (2 \omega+\phi-\theta) & \cdots & \cos (M \omega+\phi-\theta)
\end{array}\right]^{T}
$$

and

$$
\tilde{\mathbf{V}}_{s}=\sqrt{\frac{2}{N}}\left[\begin{array}{llll}
\cos (\theta) & \cos (M \omega+\theta) & \cdots & \cos (M(N-1) \omega+\theta) \\
\sin (\theta) & \sin (M \omega+\theta) & \cdots & \sin (M(N-1) \omega+\theta)
\end{array}\right]^{T}
$$

where $\theta \in[0,2 \pi)$ is an arbitrary parameter. The corresponding noise-free singular values are then determined as: 


$$
\tilde{\lambda}_{1}=\tilde{\lambda}_{2}=\frac{\sqrt{M N} \gamma}{2}
$$

To estimate $\omega$ from $\mathbf{U}_{s}$, we first let $\alpha=2 \cos (\omega)$. According to the linear prediction property of $s_{k}+s_{k-2}=\alpha s_{k-1}$, we construct:

$$
\mathbf{c} \alpha \approx \mathbf{d}
$$

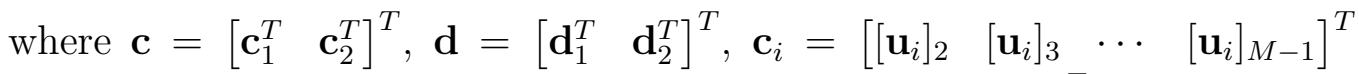
and $\mathbf{d}_{i}=\left[\begin{array}{llll}{\left[\mathbf{u}_{i}\right]_{1}+\left[\mathbf{u}_{i}\right]_{3}} & {\left[\mathbf{u}_{i}\right]_{2}+\left[\mathbf{u}_{i}\right]_{4}} & \cdots & {\left[\mathbf{u}_{i}\right]_{M-2}+\left[\mathbf{u}_{i}\right]_{M}}\end{array}\right]^{T}$ with []$_{i}$ being the $i$ th element in a vector. Employing the WLS technique, the ideal estimate of $\alpha$, denoted by $\hat{\alpha}$, is [5]:

$$
\hat{\alpha}=\frac{\mathbf{c}^{T} \mathbf{W}_{M}(\alpha) \mathbf{d}}{\mathbf{c}^{T} \mathbf{W}_{M}(\alpha) \mathbf{c}}
$$

Defining A = Toeplitz $\left(\left[\begin{array}{ll}1 & \mathbf{0}_{1 \times(M-3)}\end{array}\right]^{T},\left[\begin{array}{llll}1 & -\alpha & 1 & \mathbf{0}_{1 \times(M-3)}\end{array}\right]\right)$ where $\operatorname{Toeplitz}\left(\mathbf{a}, \mathbf{b}^{T}\right)$ is a Toeplitz matrix with first column $\mathbf{a}$ and first row $\mathbf{b}^{T}$ and $\mathbf{0}_{i \times j}$ represents an $i \times j$ zero matrix, and $\mathbf{u}_{s}=\left[\begin{array}{ll}\mathbf{u}_{1}^{T} & \mathbf{u}_{2}^{T}\end{array}\right]^{T}$, and noting that $\mathbf{A} \tilde{\mathbf{u}}_{s}=\mathbf{0}_{(M-2) \times 1}$, the optimal weighting matrix $\mathbf{W}_{M}(\alpha)$, which is characterized by the unknown $\alpha$, is computed as:

$$
\begin{aligned}
\mathbf{W}_{M}(\alpha) & =\sigma^{2}\left[\mathbb{E}\left\{(\mathbf{c} \alpha-\mathbf{d})(\mathbf{c} \alpha-\mathbf{d})^{T}\right\}\right]^{-1} \\
& =\sigma^{2}\left[\mathbb{E}\left\{\mathbf{A} \mathbf{u}_{s} \mathbf{u}_{s}^{T} \mathbf{A}^{T}\right\}\right]^{-1} \\
& =\operatorname{diag}\left(\tilde{\lambda}_{1}^{2}, \tilde{\lambda}_{2}^{2}\right) \otimes\left(\mathbf{A} \mathbf{A}^{T}\right)^{-1} \\
& \approx \operatorname{diag}\left(\lambda_{1}^{2}, \lambda_{2}^{2}\right) \otimes\left(\mathbf{A} \mathbf{A}^{T}\right)^{-1}
\end{aligned}
$$

where $\mathbb{E}, \otimes,^{-1}$ denote the expectation operator, Kronecker product and matrix inverse, respectively. Note that the noise-free singular values are approximated by $\lambda_{1}$ and $\lambda_{2}$. As $\alpha$ is unknown, we use the following iterative relaxation procedure [5] for its determination:

(i) Obtain an initial estimate of $\alpha, \hat{\alpha}$, using (16) with $\mathbf{W}_{M}(\alpha)=\operatorname{diag}\left(\lambda_{1}^{2}, \lambda_{2}^{2}\right) \otimes$ $\mathbf{I}_{M-2}$, where $\mathbf{I}_{i}$ denotes an $i \times i$ identity matrix.

(ii) Construct (17) using $\alpha=\hat{\alpha}$.

(iii) Compute an updated $\hat{\alpha}$ using (16).

(iv) Repeat Steps (ii)-(iii) until a stopping criterion is reached. 
(v) The frequency estimate based on $\mathbf{U}_{s}$, denoted by $\hat{\omega}_{L}$, is calculated as:

$$
\hat{\omega}_{L}=\cos ^{-1}\left(\frac{\hat{\alpha}}{2}\right)
$$

Let $\beta=2 \cos (M \omega)$. In a similar manner, the conceptual solution for $\beta$ obtained from $\mathbf{V}_{s}$ is

$$
\hat{\beta}=\frac{\mathbf{e}^{T} \mathbf{W}_{N}(\beta) \mathbf{f}}{\mathbf{e}^{T} \mathbf{W}_{N}(\beta) \mathbf{e}}
$$

where

$$
\mathbf{W}_{N}(\beta) \approx \operatorname{diag}\left(\lambda_{1}^{2}, \lambda_{2}^{2}\right) \otimes\left(\mathbf{B B}^{T}\right)^{-1}
$$

with $\mathbf{B}=\operatorname{Toeplitz}\left(\left[\begin{array}{ll}1 & \mathbf{0}_{1 \times(N-3)}\end{array}\right]^{T},\left[\begin{array}{llll}1 & -\beta & 1 & \mathbf{0}_{1 \times(N-3)}\end{array}\right]\right), \mathbf{e}=\left[\begin{array}{ll}\mathbf{e}_{1}^{T} & \mathbf{e}_{2}^{T}\end{array}\right]^{T}$,

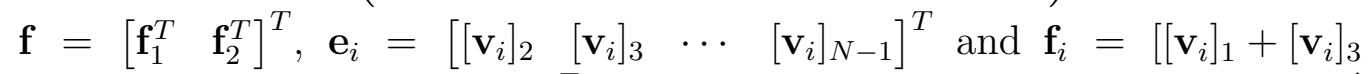
$\left.\left[\mathbf{v}_{i}\right]_{2}+\left[\mathbf{v}_{i}\right]_{4} \cdots\left[\mathbf{v}_{i}\right]_{N-2}+\left[\mathbf{v}_{i}\right]_{N}\right]^{T}$. To estimate $\omega$ using (19), we notice that $\hat{\beta}$ corresponds to $M$ possible estimates of $\omega$, denoted by $\hat{\omega}_{R, i}, i=1,2, \cdots, M$ :

$$
\hat{\omega}_{R, i}=\frac{1}{M}\left[(-1)^{(i-1)} \cos ^{-1}(\hat{\beta} / 2)+\left\lfloor\frac{i}{2}\right\rfloor 2 \pi\right]
$$

where \lfloor\rfloor rounds the value to the nearest integer towards $-\infty$. In this study, we calculate the absolute difference between $\hat{\omega}_{L}$ and each of $\left\{\hat{\omega}_{R, i}\right\}$, and the frequency estimate according to $\hat{\beta}$, denoted by $\hat{\omega}_{R}$, is given by the latter with the smallest difference. Mathematically, $\hat{\omega}_{R}=\hat{\omega}_{R, k}$ where $k$ is obtained from

$$
k=\arg \min _{i \in\{1,2, \cdots, M\}}\left|\hat{\omega}_{R, i}-\hat{\omega}_{L}\right|
$$

The variances of $\hat{\alpha}$ and $\hat{\beta}$, denoted by $\operatorname{var}(\hat{\alpha})$ and $\operatorname{var}(\hat{\beta})$, under sufficiently large $M$ and $N$, are [5]

$$
\begin{aligned}
& \operatorname{var}(\hat{\alpha}) \approx \frac{\sigma^{2}}{\tilde{\mathbf{c}}^{T} \mathbf{W}_{M}(\alpha) \tilde{\mathbf{c}}} \\
& \operatorname{var}(\hat{\beta}) \approx \frac{\sigma^{2}}{\tilde{\mathbf{e}}^{T} \mathbf{W}_{N}(\beta) \tilde{\mathbf{e}}}
\end{aligned}
$$

Employing $\alpha=2 \cos (\omega)$ and $\beta=2 \cos (M \omega)$, the variances of $\hat{\omega}_{L}$ and $\hat{\omega}_{R}$ are computed as [5]:

$$
\begin{aligned}
& \operatorname{var}\left(\hat{\omega}_{L}\right) \approx \frac{\operatorname{var}(\hat{\alpha})}{4 \sin ^{2}(\omega)} \approx \frac{\sigma^{2}}{4 \sin ^{2}(\omega) \tilde{\mathbf{c}}^{T} \mathbf{W}_{M}(\alpha) \tilde{\mathbf{c}}} \\
& \operatorname{var}\left(\hat{\omega}_{R}\right) \approx \frac{\operatorname{var}(\hat{\beta})}{4 M^{2} \sin ^{2}(M \omega)} \approx \frac{\sigma^{2}}{4 M^{2} \sin ^{2}(M \omega) \tilde{\mathbf{e}}^{T} \mathbf{W}_{N}(\beta) \tilde{\mathbf{e}}}
\end{aligned}
$$


Apparently, (26) is smaller than (25) because the former has a factor of $1 / M^{2}$. In fact, we have shown in the Appendix that

$$
\operatorname{var}\left(\hat{\omega}_{R}\right) \approx \frac{24 \sigma^{2}}{K^{3} \gamma^{2}}
$$

which approaches the asymptotic CRLB when $K \rightarrow \infty$, namely, $24 \sigma^{2} /\left(\gamma^{2} K\left(K^{2}-\right.\right.$ 1)) [1]. As a result, it is sufficient to employ $\hat{\omega}_{R}$ as the final frequency estimate. Nevertheless, we can combine $\hat{\omega}_{R}$ and $\hat{\omega}_{L}$ by assuming that they are uncorrelated, to produce the estimate $\hat{\omega}$ as:

$$
\begin{aligned}
\hat{\omega} & =\frac{\frac{\hat{\omega}_{L}}{\operatorname{var}\left(\hat{\omega}_{L}\right)}+\frac{\hat{\omega}_{R}}{\operatorname{var}\left(\hat{\omega}_{R}\right)}}{\frac{1}{\operatorname{var}\left(\hat{\omega}_{L}\right)}+\frac{1}{\operatorname{var}\left(\hat{\omega}_{R}\right)}} \\
& \approx \frac{\hat{\omega}_{L} \sin ^{2}(\hat{\omega}) \mathbf{c}^{T} \mathbf{W}_{M}(\hat{\alpha}) \mathbf{c}+\hat{\omega}_{R} M^{2} \sin ^{2}(M \hat{\omega}) \mathbf{e}^{T} \mathbf{W}_{N}(\hat{\beta}) \mathbf{e}}{\sin ^{2}(\hat{\omega}) \mathbf{c}^{T} \mathbf{W}_{M}(\hat{\alpha}) \mathbf{c}+M^{2} \sin ^{2}(M \hat{\omega}) \mathbf{e}^{T} \mathbf{W}_{N}(\hat{\beta}) \mathbf{e}}
\end{aligned}
$$

where the noise-free and ideal parameters in (25)-(26) are substituted by the noisy or estimated values.

\section{Numerical Examples}

Computer simulations have been carried out to evaluate the frequency estimation performance of the proposed estimator by comparing with the WLS [5], ML [8] and ARMA [7] approaches. Three iterations are employed as the stopping criterion in the WLS and proposed methods because no significant improvement is observed for more iterations while the golden section search is used for computing the ML solution after a discrete Fourier transform style coarse estimate is obtained. The mean square error (MSE) is assigned as the performance measure and CRLB is also included to validate the algorithm optimality. The sinusoidal amplitude is chosen as $\gamma=1$ and we properly scale the zero-mean white Gaussian noise sequences $\left\{q_{k}\right\}$ to produce different SNR conditions, where SNR $=\gamma^{2} /\left(2 \sigma^{2}\right)=1 /\left(2 \sigma^{2}\right)$. All results provided are averages of 1000 independent runs using a computer with Intel Core i7 $2.67 \mathrm{GHz}$ processors and 3GB RAM.

In the first test, the performance of the proposed scheme based on $\hat{\omega}_{R}$ and $\hat{\omega}$ of (28) is compared. Figure 1 shows their MSEs versus SNR at $K=256$ and $K=1024$ with $M=N=16$ and $M=N=32$, respectively. The tone 
frequency is $\omega=0.1 \pi$ while the phase $\phi$ is uniformly chosen from $[0,2 \pi)$ for each independent trial. It is seen that both $\hat{\omega}_{R}$ and $\hat{\omega}$ perform almost the same for $K=256$ and $K=1024$, and their accuracy attains the CRLB for sufficiently high SNR conditions. The corresponding threshold SNRs are $0 \mathrm{~dB}$ and $-4 \mathrm{~dB}$, indicating that a larger $K$ gives a better threshold performance. According to Figure 1, only $\hat{\omega}_{R}$ is considered in the remaining experiments.

Figures 2 and 3 investigate different combinations of $M$ and $N$ for $K=$ 256 and $K=1024$, respectively. The other parameter settings are identical to those of Figure 1. Although the MSEs of different combinations can attain optimality for sufficiently high SNRs, we should avoid $M \ll N$ or $M \gg N$ because these correspond to a larger threshold SNR. For $K=256$, the average computation times of the proposed estimator with $(M, N)$ equals $(4,64),(8,32),(16,16),(32,8)$ and $(64,4)$ are $0.0023 \mathrm{~s}, 0.0014 \mathrm{~s}, 0.0013 \mathrm{~s}$, $0.0014 \mathrm{~s}, 0.0024 \mathrm{~s}$, respectively. The corresponding measured times for $(8,128)$, $(16,64),(32,32),(64,16)$ and $(128,8)$ are $0.0086 \mathrm{~s}, 0.0031 \mathrm{~s}, 0.0027 \mathrm{~s}, 0.0032 \mathrm{~s}$, 0.0092s. Summarizing the results, the best combination in terms of estimation performance and computational complexity is $M \approx N$.

In the remaining tests, the proposed method is compared with the WLS, ML and ARMA approaches. Figure 4 shows the MSEs versus SNR at $K=$ 256. The parameter settings are identical to those of Figure 1. The proposed, WLS, ML and ARMA estimators have threshold SNRs of $0 \mathrm{~dB}, 10 \mathrm{~dB},-6 \mathrm{~dB}$ and $6 \mathrm{~dB}$, while their average computation times are measured as $0.0013 \mathrm{~s}$, $0.0147 \mathrm{~s}, 0.0086 \mathrm{~s}$ and $0.0002 \mathrm{~s}$. Although the ARMA method is the most computationally efficient, it produces biased estimation when $\mathrm{SNR} \geq 16 \mathrm{~dB}$. On the other hand, the ML estimator has the best threshold performance but its complexity is higher than that of the proposed scheme. The MSEs of different methods versus $\omega \in(0, \pi)$ at $\mathrm{SNR}=10 \mathrm{~dB}$ are plotted in Figure 5. Similar to the ML and WLS algorithms, the proposed method also has uniform estimation performance when $\omega$ is not close to 0 or $\pi$.

\section{Conclusion}

A fast and accurate estimator for a single real tone based on singular value decomposition of the corresponding data matrix has been devised. The techniques of linear prediction and weighted linear squares (WLS) are utilized in the principal singular vectors which contain the frequency information, for parameter estimation. It is proved that the performance of the estimator attains Cramér-Rao lower bound at sufficiently large data lengths. Further- 
more, it is demonstrated that the proposed method is superior to the WLS, maximum likelihood and autoregressive moving average model fitting methods in terms of accuracy and/or computational complexity.

\section{Appendix}

In this Appendix, we prove that var $\left(\hat{\omega}_{R}\right)$ attains the CRLB when $K \rightarrow \infty$. With the use of (13) and (14), the asymptotic value of $\operatorname{var}(\hat{\beta})$ of $(24)$ is derived as

$$
\begin{aligned}
\operatorname{var}(\hat{\beta}) & \approx \frac{\sigma^{2}}{\tilde{\mathbf{e}}^{T} \mathbf{W}_{N}(\beta) \tilde{\mathbf{e}}}=\frac{\sigma^{2}}{\sum_{i=1}^{2} \tilde{\lambda}_{i}^{2} \tilde{\mathbf{e}}_{i}^{T}\left(\mathbf{B B}^{T}\right)^{-1} \tilde{\mathbf{e}}_{i}} \\
& \approx \frac{4 \sigma^{2}}{M N \gamma^{2} \sum_{i=1}^{2} \tilde{\mathbf{e}}_{i}^{T}\left(\mathbf{B B}^{T}\right)^{-1} \tilde{\mathbf{e}}_{i}}
\end{aligned}
$$

where $\left[\tilde{\mathbf{e}}_{1}\right]_{k}=\cos (M k \omega+\theta),\left[\tilde{\mathbf{e}}_{2}\right]_{k}=\sin (M k \omega+\theta), k=1,2, \cdots, N-2$. When $N$ is sufficiently large, it is shown that [5]:

$$
\frac{1}{\tilde{\mathbf{e}}_{1}^{T}\left(\mathbf{B B}^{T}\right)^{-1} \tilde{\mathbf{e}}_{1}} \approx \frac{1}{\tilde{\mathbf{e}}_{2}^{T}\left(\mathbf{B B}^{T}\right)^{-1} \tilde{\mathbf{e}}_{2}} \approx \frac{96 \sin ^{2}(M \omega)}{(N-4)\left((N-4)^{2}-1\right)} \approx \frac{96 \sin ^{2}(M \omega)}{N^{3}}
$$

Substituting (A.2) into (A.1) yields

$$
\operatorname{var}(\hat{\beta}) \approx \frac{96 \sigma^{2} \sin ^{2}(M \omega)}{M N^{3} \gamma^{2}}
$$

As a result, (26) is:

$$
\operatorname{var}\left(\hat{\omega}_{R}\right) \approx \frac{\operatorname{var}(\hat{\beta})}{4 M^{2} \sin ^{2}(M \omega)} \approx \frac{24 \sigma^{2}}{K^{3} \gamma^{2}}
$$

which is $(27)$.

\section{References}

[1] S. M. Kay, Fundamentals of Statistical Signal Processing: Estimation Theory, Upper Saddle River, NJ: Prentice-Hall, 1993

[2] P. Stoica and R. Moses, Spectral Analysis of Signals, Upper Saddle River, NJ : Prentice-Hall, 2005 
[3] V. F. Pisarenko, "The retrieval of harmonics by linear prediction," Geophys. J. Roy. Astron. Soc., vol.33, pp.347-366, 1973

[4] H. C. So and K. W. Chan, "Reformulation of Pisarenko harmonic decomposition method for single-tone frequency estimation," IEEE Transactions on Signal Processing, vol.52, no.4, pp.1128-1135, Apr. 2004

[5] H. C. So, K. W. Chan, Y. T. Chan and K. C. Ho, "Linear prediction approach for efficient frequency estimation of multiple real sinusoids: algorithms and analyses," IEEE Transactions on Signal Processing, vol.53, no.7, pp.22902305, Jul. 2005

[6] R. Elasmi-Ksibi, S. Cherif, R. Lopez-Valcarce and H. Besbes, "Closed-form real single-tone frequency estimator based on a normalized IIR notch filter," Signal Processing, vol.90, pp.1905-1915, 2010

[7] B. G. Quinn and J. M. Fernandes, "A fast efficient technique for the estimation of frequency," Biometrika, vol.78, no.3, pp.489-497, Sep. 1991

[8] R. J. Kenefic and A. H. Nuttall, "Maximum likelihood estimation of the parameters of tone using real discrete data," IEEE Journal of Oceanic Engineering, vol.12, no.1, pp.279-280, 1987

[9] A. Saucier, "New periodograms for single-tone frequency estimation in the presence of an additive polynomial background signal," Signal Processing, vol.90, pp.1800-1814, 2010

[10] H. W. Fung, A. C. Kot, K. H. Li and K. C. Teh, "Parameter estimation of a real single tone from short data records," Signal Processing, vol.84, pp.601617, 2004

[11] I. Djurovic and V. V. Lukin, "Estimation of single-tone signal frequency by using the L-DFT," Signal Processing, vol.87, pp.1537-1544, 2007

[12] K. W. K. Lui and H. C. So, "Two-stage autocorrelation approach for accurate single sinusoidal frequency estimation," Signal Processing, vol.88, no.7, pp.1852-1857, Jul. 2008

[13] R. Elasmi-Ksibi, H. Besbes, R. Lopez-Valcarce and S. Cherif, "Frequency estimation of real-valued single-tone in colored noise using multiple autocorrelation lags," Signal Processing, vol.90, pp.2303-2307, 2010 
[14] H. C. So, F. K. W. Chan, W. H. Lau and C. F. Chan, "An efficient approach for two-dimensional parameter estimation of a single-tone," IEEE Transactions on Signal Processing, vol.58, no.4, pp.1999-2009, Apr. 2010

[15] H. C. So, F. K. W.Chan and W. Sun, "Subspace approach for fast and accurate single-tone frequency estimation," IEEE Transactions on Signal Processing, vol.59, no.2, pp.827-831, Feb. 2011

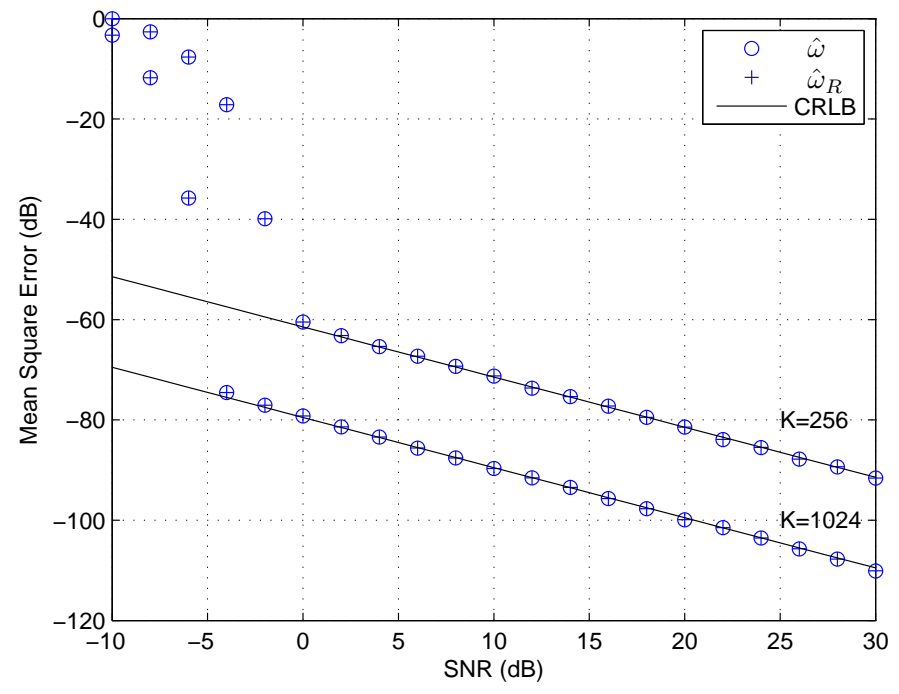

Figure 1: Mean square frequency error versus SNR for different $K$ 


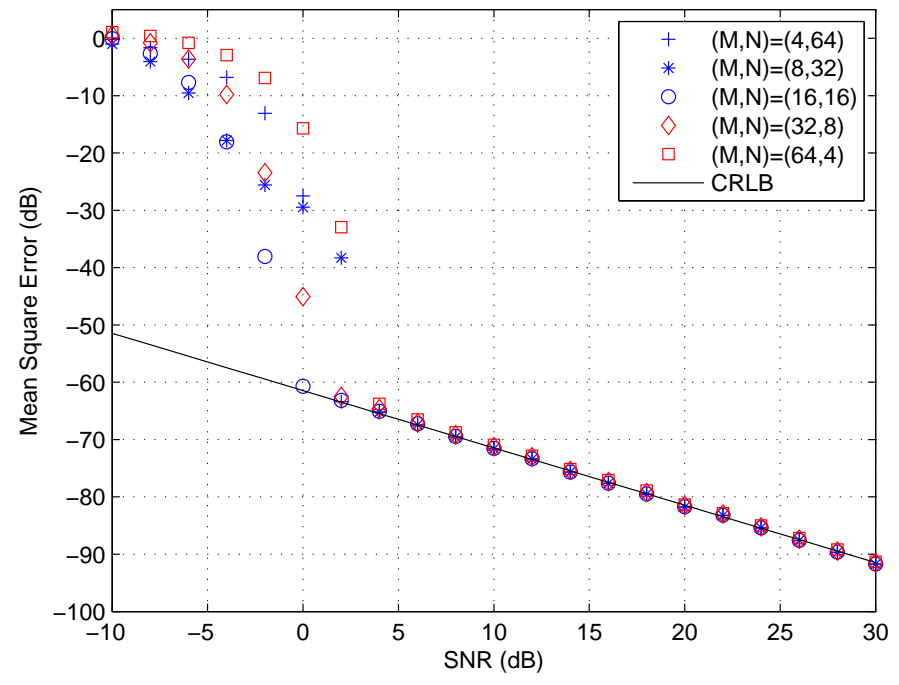

Figure 2: Mean square frequency error versus SNR for different combinations of $(M, N)$ at $K=256$

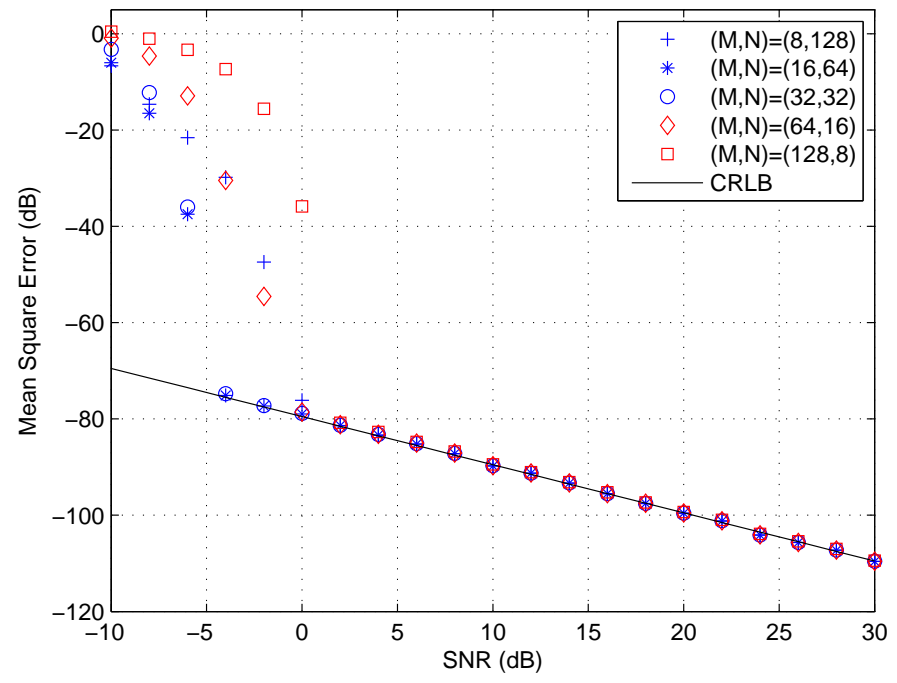

Figure 3: Mean square frequency error versus SNR for different combinations of $(M, N)$ at $K=1024$ 


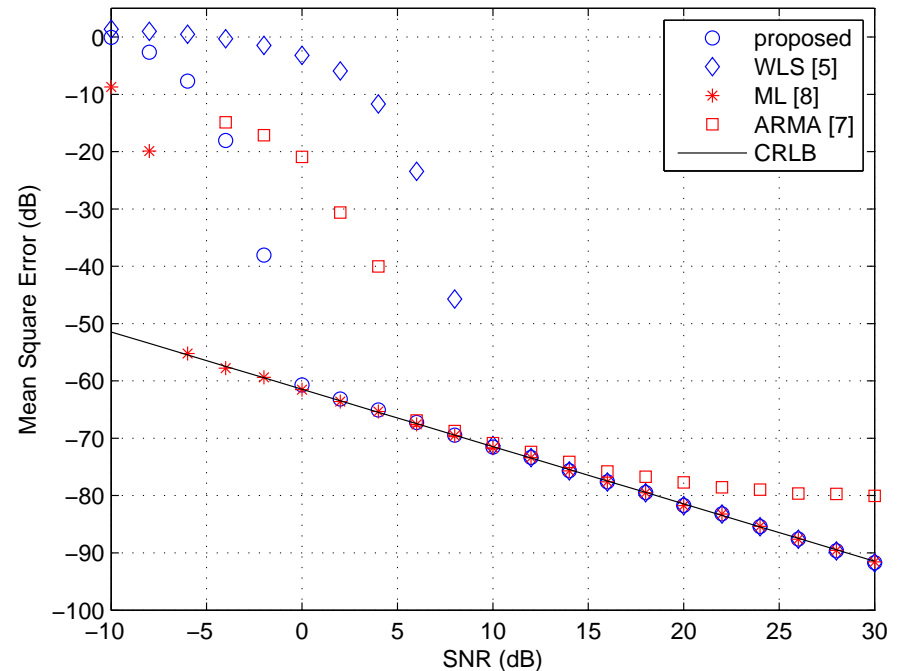

Figure 4: Mean square frequency errors versus SNR at $K=256$

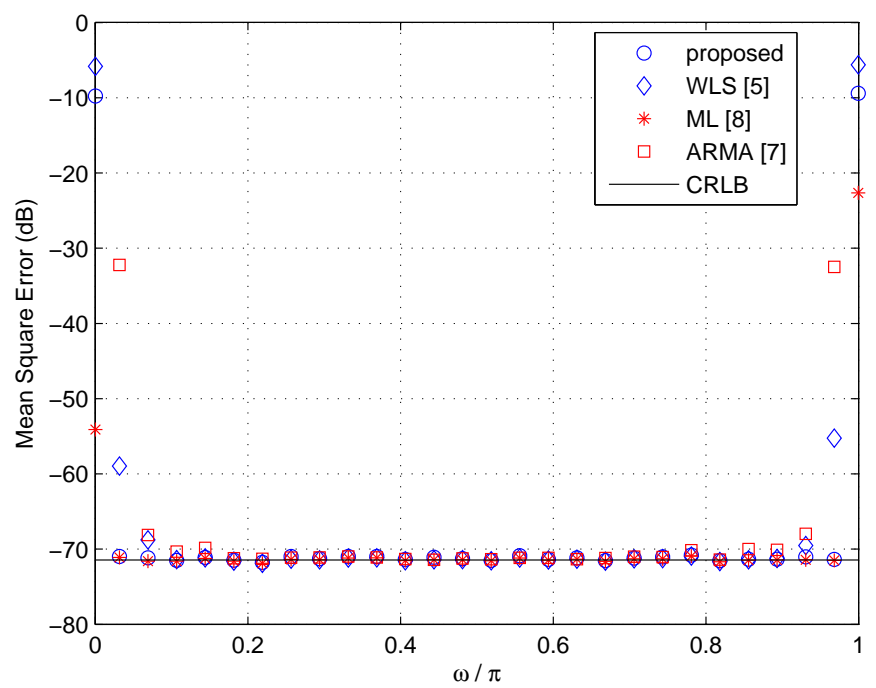

Figure 5: Mean square frequency errors versus $\omega \in(0, \pi)$ at $\mathrm{SNR}=10 \mathrm{~dB}$ 